\title{
Complete Genome Sequence Data of Tumorigenic Rhizobium vitis Strain VAT03-9, a Causal Agent of Grapevine Crown Gall Disease
}

\author{
Yoshiteru Noutoshi, ${ }^{1, \dagger}$ Atsushi Toyoda, ${ }^{2}$ Tomoya Ishii, ${ }^{1}$ Kirara Saito, ${ }^{1}$ Megumi Watanabe, ${ }^{1}$ and \\ Akira Kawaguchi ${ }^{3}$ \\ ${ }^{1}$ Graduate School of Environmental and Life Science, Okayama University, Okayama, Japan \\ ${ }^{2}$ Department of Genomics and Evolutionary Biology, National Institute of Genetics, Mishima, Shizuoka, \\ Japan \\ ${ }^{3}$ Western Region Agricultural Research Center, National Agricultural and Food Research Organization \\ (NARO), Fukuyama, Hiroshima, Japan
}

\begin{abstract}
Rhizobium vitis strain VAT03-9 (MAFF 211676) is a causal agent of crown gall disease in grapevine. It is one of the pathogenic strains of $R$. vitis isolated from graft unions of grapevine in Okayama Prefecture, Japan. Inoculation tests verified its virulence for gall formation on grapevine, tomato, and sunflower. It harbors tumor-inducing plasmid. Here, we present the complete genome sequence with annotation of $R$. vitis VAR03-9 obtained by assembling reads from PacBio and Illumina-sequencers. This genome sequence should be useful for the analyses of pathogenicity and evolutionary lineage of the pathogens of crown gall disease.
\end{abstract}

\section{Funding}

This work was financially supported by Japan Society for the Promotion of Science grant numbers KAKENHI 17H03778 and KAKENHI $20 \mathrm{~K} 20572$ to

A. Kawaguchi and Y. Noutoshi and KAKENHI 16H06279 (Platform for Advanced Genome Science) from the Ministry of Education, Culture, Sports, Science and Technology of Japan.

\section{Keywords}

bacterial pathogenesis, crown gall disease, grapevine, Rhizobium vitis, tumor-inducing plasmid

\section{Genome Announcement}

Rhizobium vitis (=Agrobacterium vitis, A. radiobacter biovar 3 ) strain VAR03-9 (MAFF211676) was isolated from graft unions of nursery stock of grapevine (Vitis vinifera L. 'Seto Giants') in a field of Okayama Prefecture, Japan using Roy and Sasser's medium, a semiselective medium for $R$. vitis (Kawaguchi et al. 2005; Roy and Sassar 1983). In this article, the nomenclature for Rhizobium and Agrobacterium spp. follows a proposal by Young et al. (2001). The characteristics of classical diagnostic tests with cultural, biochemical, and physiological properties of this strain were coincided with those of $R$. vitis $(=A$. vitis, Agrobacterium spp. biovar 3). This result was also supported by a multiplex PCR analysis using primer pairs which can amplify the virC gene involved in tumorigenesis or rhizogenesis of Agrobacterium and Rhizobium strains and 16S ribosomal DNA of $R$. vitis (Kawaguchi et al. 2005). Consistent with the detection of the virulence-related gene, its gall formation activity was demonstrated on grapevine. In addition, its pathogenicity was also observed on sunflower and tomato.

Crown gall disease on grapevine is an important disease in the world and it causes serious damage in fruit yields and wine production. Its causal agent was originally known as A. tumefaciens (Smith \& Townsend 1907) Conn 1942. The diagnostic characteristics of this

${ }^{\dagger}$ Corresponding author: Y. Noutoshi; noutoshi@okayama-u.ac.jp

Present address of K. Saito: Kyusyu Okinawa Agricultural Research Center, National Agriculture and Food Research Organization, Miyakonojo, Miyazaki, Japan.

The author(s) declare no conflict of interest.

Accepted for publication 11 August 2020. 
pathogen were shown to be different from those of $A$. tumefaciens and $A$. rhizogenes, which are classified as biovar 1 and 2, respectively; therefore, it was newly categorized as biovar 3 of Agrobacterium. Agrobacterium and Rhizobium spp. were used for pathogenic and symbiotic strains, respectively, but today they are considered to be the same clade. The pathogenic bacterial strains isolated from grapevine crown gall disease are now classified as tumorigenic $R$. vitis. The pathogenicity of Agrobacterium and Rhizobium relies on the transmittable tumor-inducing (Ti) or root-inducing plasmid.

The whole-genome sequence of $R$. vitis (=A. vitis) S4 has been reported thus far with annotation (Slater et al. 2009). It is a virulent strain on grapevine found in Europe and was known to induce a hypersensitive response in nonhost plants. The genome sequences in the other Rhizobium bacteria have been reported in tumorigenic $R$. radiobacter (=tumorigenic $A$. tumefaciens, $A$. tumefaciens biovar 1 ) strain C58 and nonpathogenic $R$. rhizogenes (=nonpathogenic $A$. rhizogenes, $A$. radiobacter biovar 2) strain $\mathrm{K} 84$, and organization of the genomes in these strains was compared (Goodner et al. 2001; Slater et al. 2009; Wood et al. 2001).

In this study, we report whole-genome sequencing and annotation of $R$. vitis VAT03-9 as another strain of $R$. vitis by using PacBio and Illumina sequencing platforms. The genomic DNA was isolated using a QIAamp DNA Mini Kit (Qiagen, Hilden, Germany) and it was sheared into fragments ranging from 20 to $50 \mathrm{kbp}$ in size with a g-tube device (Covaris Inc., Woburn, MA, U.S.A.). Then, we prepared a SMRTbell library with a Template Prep Kit v1.0 (Pacific Bioscience, Menlo Park, CA, U.S.A.) according to the manufacturer's protocol. The size of the sequencing library was selected using the BluePippin system (Sage Science, Beverly, MA, U.S.A.) with a minimum fragment length cutoff of $17 \mathrm{kbp}$. The sequence reads were then obtained from the resultant library by the PacBio RSII sequencer with SMRT cell v3 and P6-C4v2 chemistry, yielding a total of 70,337 reads (1.65 Gbp). These reads were assembled using the HGAP3 assembler program with default settings. In parallel, genomic DNA was sheared into fragments with an average size of 600 bp by the DNA Shearing System M220 (Covaris Inc.). A paired-end library was made with a TruSeq DNA PCR-Free Library Prep kit (Illumina, Inc., San Diego, CA, U.S.A.) and its size was selected on an agarose gel using a Zymoclean Large Fragment DNA Recovery Kit (Zymo Research, Irvine, CA, U.S.A.). The resultant paired-end library was run on the Illumina HiSeq 2500 sequencer with a read length of $250 \mathrm{bp}$. To improve the base quality of the long-read assembly, Illumina read sequences comprising 3,186,764 $\times 2$ raw reads were processed with the Pilon version 1.22 software tool. For annotation, a DNA Data Bank of Japan (DDBJ) Fast Annotation and Submission Tool (DFAST) was used (Tanizawa et al. 2016).

The total genome size of tumorigenic $R$. vitis VAT03-9 is 6,223,633 bp in length without any gaps and its GC content is $57.5 \%$. In total, there were 5,700 protein-coding sequences. The genome is divided into two chromosomes (circular) whose sizes are $3,717,176$ and $1,132,123$ bp and five plasmids whose sizes are 609,097, 371,025, 257,173, 88,753, and 48,286 bp. Nine ribosomal RNAs and 52 transfer RNAs are located on chromosome 1, and three ribosomal RNAs and five transfer RNAs exist on chromosome 2 . The virulence genes such as virB, virE, and virD and T-DNA conjugal transfer-related genes are located on the 257,173-bp-length plasmid which contains 265 genes in total. This is thought to be a Ti plasmid of this strain.

This genome sequence should be useful for the analyses of pathogenicity and evolutionary lineage of the pathogens of crown gall disease.

This complete whole-genome sequence has been deposited in the DDBJ database under accession numbers AP023279 (chr1), AP023280 (chr2), AP023281 (pRvVAR031a), AP023282 (pRvVAR031b), AP023283 (pTiRvVAT039), AP023284 (pRvVAR031c), and AP023285 (pRvVAR031d) (PRJDB10104 for BioProject and SAMD00233296 for BioSample).

\section{Acknowledgments}

We thank the Platform for Advanced Genome Science and its scientific and supporting members for sequencing, financial support, lectures, and management. 


\section{Literature Cited}

Goodner, B., Hinkle, G., Gattung, S., Miller, N., Blanchard, M., Qurollo, B., Goldman, B. S., Cao, Y., Askenazi, M., Halling, C., Mullin, L., Houmiel, K., Gordon, J., Vaudin, M., lartchouk, O., Epp, A., Liu, F., Wollam, C., Allinger, M., Doughty, D., Scott, C., Lappas, C., Markelz, B., Flanagan, C., Crowell, C., Gurson, J., Lomo, C., Sear, C., Strub, G., Cielo, C., and Slater, S. 2001. Genome sequence of the plant pathogen and biotechnology agent Agrobacterium tumefaciens C58. Science 294:2323-2328.

Kawaguchi, A., Inoue, K., and Nasu, H. 2005. Inhibition of crown gall formation by Agrobacterium radiobacter biovar 3 strains isolated from grapevine. J. Gen. Plant Pathol. 71:422-430.

Roy, M. A., and Sassar, M. 1983. A medium selective for Agrobacterium tumefaciens biotype 3. (Abstr.) Phytopathology 73:810.

Slater, S. C., Goldman, B. S., Goodner, B., Setubal, J. C., Farrand, S. K., Nester, E. W., Burr, T. J., Banta, L., Dickerman, A. W., Paulsen, I., Otten, L., Suen, G., Welch, R., Almeida, N. F., Arnold, F., Burton, O. T., Du, Z., Ewing, A., Godsy, E., Heisel, S., Houmiel, K. L., Jhaveri, J., Lu, J., Miller, N. M., Norton, S., Chen, Q., Phoolcharoen, W., Ohlin, V., Ondrusek, D., Pride, N., Stricklin, S. L., Sun, J., Wheeler, C., Wilson, L., Zhu, H., and Wood, D. W. 2009. Genome sequences of three agrobacterium biovars help elucidate the evolution of multichromosome genomes in bacteria. J. Bacteriol. 191:2501-2511.
Tanizawa, Y., Fujisawa, T., Kaminuma, E., Nakamura, Y., and Arita, M. 2016. DFAST and DAGA: Web-based integrated genome annotation tools and resources. Biosci. Microbiota Food Health 35:173-184.

Wood, D. W., Setubal, J. C., Kaul, R., Monks, D. E., Kitajima, J. P., Okura, V. K., Zhou, Y., Chen, L., Wood, G. E., Almeida, N. F., Jr., Woo, L., Chen, Y., Paulsen, I. T., Eisen, J. A., Karp, P. D., Bovee, D., Sr., Chapman, P., Clendenning, J., Deatherage, G., Gillet, W., Grant, C., Kutyavin, T., Levy, R., Li, M. J., McClelland, E., Palmieri, A., Raymond, C., Rouse, G., Saenphimmachak, C., Wu, Z., Romero, P., Gordon, D., Zhang, S., Yoo, H., Tao, Y., Biddle, P., Jung, M., Krespan, W., Perry, M., Gordon-Kamm, B., Liao, L., Kim, S., Hendrick, C., Zhao, Z. Y., Dolan, M., Chumley, F., Tingey, S. V., Tomb, J. F., Gordon, M. P., Olson, M. V., and Nester, E. W. 2001. The genome of the natural genetic engineer Agrobacterium tumefaciens C58. Science 294:2317-2323.

Young, J. M., Kuykendall, L. D., Martínez-Romero, E., Kerr, A., and Sawada, H. 2001. A revision of Rhizobium Frank 1889, with an emended description of the genus, and the inclusion of all species of Agrobacterium Conn 1942 and Allorhizobium undicola de Lajudie et al. 1998 as new combinations: Rhizobium radiobacter, $R$. rhizogenes, $R$. rubi, $R$. undicola and $R$. vitis. Int. J. Syst. Evol. Microbiol. 51:89-103. 